\title{
The Expression Discrepancy And Characteristics of Long Non-Coding RNAs In Peripheral Blood Leukocytes From Amyotrophic Lateral Sclerosis Patients
}

\section{Yujiao Yu}

Sichuan University West China Hospital

\section{Dejiang Pang}

Sichuan University West China Hospital

\section{Chunyu Li}

Sichuan University West China Hospital

\section{Xiaojing Gu}

Sichuan University West China Hospital

\section{Yongping Chen}

Sichuan University West China Hospital

\section{Ruwei Ou}

Sichuan University West China Hospital

Qianqian Wei

Sichuan University West China Hospital

Huifang Shang ( $\nabla$ hfshang2002@126.com )

Sichuan University West China Hospital https://orcid.org/0000-0003-0947-1151

\section{Research Article}

Keywords: LncRNA, Amyotrophic lateral sclerosis, biomarkers, neurodegenerative disease

Posted Date: July 23rd, 2021

DOI: https://doi.org/10.21203/rs.3.rs-565200/v2

License: (c) (1) This work is licensed under a Creative Commons Attribution 4.0 International License.

Read Full License

Version of Record: A version of this preprint was published at Molecular Neurobiology on April 2nd, 2022. See the published version at https://doi.org/10.1007/s12035-022-02789-4. 


\section{Abstract}

Amyotrophic lateral sclerosis (ALS) is known to be a progressive neurodegenerative disease that affects upper and lower motor neurons. Less than $10 \%$ of ALS patients are defined as familial ALS, more than $90 \%$ are sporadic ALS (SALS). According to the genomic information described in existing databases, up to $98 \%$ of the human genome consists of non-coding sequences. Nearly $40 \%$ of long non-coding RNAs (IncRNAs) are specifically expressed in the brain. We believe that the discrepancy of IncRNAs expression plays a key role in neurodegenerative diseases.

We screened 30 IncRNAs with altered expression from peripheral blood leukocytes of SALS patients by microarray and validated 13 of them in leukocytes of SALS, Parkinson's disease (PD) patients and healthy controls $(\mathrm{HC})$. We followed the bioinformatics to perform a functional enrichment analysis of coexpressed mRNAs, transcription factors and IncRNAs for functional prediction. We identified Inc-DYRYK27:1, Inc-ABCA12-3:1 and Inc-POTEM-4:7 show decreased expression in SALS patients, whereas in PD patients they show increased expression or no change. In addition, expression of Inc-CNTN4-2:1 and IncNR3C2-8:1 were decreased in both SALS and PD patients. We found that XIST was only reduced in male patients with SALS and PD, and not in female patients with SALS but was elevated in PD by gender grouping. We also performed GO term enrichment and KEGG pathway analysis for IncRNAs showing differential expression in microarray. We discovered that a significant proportion of differential expressed IncRNAs were associated with various signaling pathways and transcription factors which are consistent with other clinical findings.

\section{Introduction}

Amyotrophic lateral sclerosis (ALS) is comprehended as one type of progressive neurodegenerative disease affecting both upper and lower motor neurons. ALS results in muscle weakness, cognitive or behavioral change and eventual death from respiratory insufficiency within 3-5 years after diagnosis[Citation error]. Less than $10 \%$ of ALS patients are hereditary with mainly dominant inheritance, which is defined as familial ALS [1, 2]. Hence, the rest of the proportion that more than $90 \%$ is sporadic ALS (SALS) [3, 4]. Recent studies have revealed that abnormalities in RNA processing and metabolism play an important role in the pathogenesis of ALS [5,6]. Molecular genetic studies have identified several ALS causative genes, such as the TARDBP gene (encoding the TDP-43 protein) [7], the FUS/TLS gene (encoding the FUS protein) [8], the ANG gene (encoding the Angiogenin protein) [9], and the C9ORF72 gene (encoding the C9ORF72 protein) [10], which are The encoded DNA/RNA-binding proteins are involved in RNA processing and metabolic processes.

Based on genome information described by the ENCODE project, up to $98 \%$ human genome is composed of a non-coding sequence [11]. Noncoding RNA (ncRNA) is one type of RNA sequence which is lacking an open reading frame that is not able to code proteins. In actuality, with the advance of biotechnology in recent years, these ncRNAs were perceived to associate with some biochemical activities including various cellular regulation and even relate to disease progress [12-14]. The classification of ncRNAs is 
usually defined by their length or function. NcRNAs length longer than 200 nucleotides are regarded as long non-coding RNAs (LncRNAs), while others are microRNAs (miRNAs), small interfering RNAs (siRNAs), small nucleolar RNAs (snoRNAs), small nuclear RNAs (snRNAs), and PIWl-interacting RNAs (piRNAs) [15-17]. The discovery of IncRNAs has provided new insights into the regulation of diseaseassociated genes. LncRNAs are thought to interact with RNA, DNA or proteins to promote or repress the expression of protein-coding genes and their function [18].

It is noteworthy that nearly $40 \%$ of IncRNAs are specifically expressed in the central nervous system (CNS) [19]. LncRNAs are heavily distributed in the CNS, which is presumably due to the complexity of CNS function, which requires a greater number of regulatory RNAs to maintain normal brain development and function. Studies have shown that IncRNAs are closely associated with neuronal differentiation, synaptogenesis, or other functional maintenance processes [20,21]. Due to the functional impact and effect of IncRNAs on a variety of biological molecules, it is reasonable to consider the role it plays in several disease conundrums. Indeed and because of that, dysregulation of IncRNA expression can play many key roles in neurodegenerative diseases. The dysregulated states of several IncRNAs displayed in neurodegenerative diseases such as Alzheimer's or Parkinson's disease, as well as their associated biochemical functions and protein or mRNA regulatory functions, have been published [22-24]. Some of these IncRNAs are thought to be potential candidate biomarkers for predicting neurodegenerative diseases [25]. Therefore, we are not surprised that IncRNAs, which occupy a large number of RNAs, have also been reported in ALS cases in recent years.

In clinical work, it typically takes a longer time to diagnose ALS disease compared to other classes of neurodegenerative diseases, and yet the condition of patients often deteriorates during this time [26]. Some biomarkers involving oxidative stress and inflammation are associated with ALS in recent years through testing of blood, plasma or serum, but diagnosis using early biomarkers is still difficult [27]. Studies on ALS have found that abnormal expression of IncRNAs is involved in the development of ALS, while functional findings also suggest that IncRNAs have an important role in the development of ALS disease. In a recent study, 293 IncRNAs and 87 mRNAs were found to be differentially expressed in the ALS group and control group by RNA sequencing in ALS patients carrying FUS, SOD1 and TARDBP mutations [28]. However, the involvement of IncRNAs in the pathogenesis of ALS was not further elucidated. There are also in-depth studies showing that some of the physiological mechanisms of IncRNA. The IncRNA named NEAT1_2 interacts with the paraspeckle formation in spinal cord motor neurons of ALS patients [29]. Nevertheless, the exact mechanisms of those published LncRNAs are not explained well yet.

It has been shown that Chinese patients with ALS have an earlier age of onset than the Caucasian population [30], and the genetic characteristics are very different from those of Caucasians. For example, C9ORF72 mutation is the most common cause of ALS in the Caucasian population [31], whereas C9ORF72 mutation is less than $1 \%$ in the Chinese sALS population [32]. Consequently, it is very important to use the resources of Chinese ALS patients to study the pathogenesis of Chinese ALS patients and carry out targeted drug development and meaningful disease diagnosis biomarkers. To manifest the 
potential biomarkers of sporadic ALS and provide a preliminary investigation of the role of IncRNA in the development of the ALS disease process, we screened IncRNAs from leukocytes of SALS patients and performed the validation of selected IncRNAs. In this paper, a transcriptome profiling of IncRNAs in peripheral leukocytes of SALS, PD and healthy controls is presented. Furthermore, the bioinformatics of these 'biomarker' IncRNAs in SALS was also investigated in the present study.

\section{Material And Methods}

\section{Subjects}

A subset of subjects (5 ALS and 5 CTR) was deep-sequenced for microarray while total of 52 SALS, 40 PD and 38 healthy controls samples were included for Real-Time PCR experiments. Those 10 samples were deeply sequenced with the approach. Although sequencing was based on a small number of samples, it also allowed us to explore the entire transcriptome of ALS patients at the level of non-coding genes and compare them to healthy controls then select an interesting subset of transcripts for further study and to perform Real-Time PCR validation. ALS patients underwent clinical and neurologic examinations based on the El Escorial revised criteria at the department of Neurology in West China Hospital (Chengdu, Sichuan province, China). The control subjects were recruited also at the department of Neurology in West China Hospital (Chengdu, Sichuan province, China). The clinical characteristics of participants in the following analysis were summarized in supplemental Table 1. The mean age of SALS, PD and controls were $51.60 \pm 2.86,54.19 \pm 11.86$, and $51.21 \pm 1.91$, respectively. The mean onset age of SALS and PD were $50.91 \pm 2.81$ and $52.79 \pm 11.68$. The mean disease duration of SALS and PD was 8.38 \pm 2.70 and $42.70 \pm 23.45$ months, respectively. There were no significant differences in the distribution of sex among SALS, PD, and healthy controls. Around $30 \%$ of SALS patients had initial symptoms in bulbar and the remaining $70 \%$ were starting from the spinal cord. The study protocol to obtain PBMC from patients and controls was approved by the West China Hospital of Sichuan University (Chengdu, China). Before being enrolled, the subjects participating in the study signed an informed consent form.

\section{Microarray}

All microarray experiments and bioinformatic support were performed by Shanghai OE Biotech CO., LTD (Shanghai, China).

\section{Leukocytes Collection and RNA Extraction}

Approximately $5 \mathrm{ml}$ of whole blood from all participants was collected through venipuncture into EDTAcontaining tubes before the start of clinical treatment in the morning. After centrifugation at $2000 \mathrm{rpm}$ for 10 minutes, the plasma was removed, and then $1 \mathrm{ml}$ of PBS was added to the remaining erythrocytewhite blood cell mixture and the mixture was carefully transferred to a $15 \mathrm{ml}$ centrifuge tube with $3 \mathrm{ml}$ of lymphocyte isolate (tbdscience Product no. LTS1077, Tianjin, China), centrifuge at $2000 \mathrm{rpm}$ for 15 minutes. Finally, the intermediate leukocyte layer of the centrifuged sample is aspirated, washed twice with PBS and stored in Trizol reagent at $-80^{\circ} \mathrm{C}$. The total RNA from PBMCs was isolated following the 
trizol RNA isolation technique. Homogenize leukocytes were ground in $1 \mathrm{ml}$ trizol reagent and the final total RNA concentration of the sample was measured using NanoDrop One (Thermo Scientific).

\section{Validation analysis by reverse transcription-quantitative real- time PCR (RT-qPCR)}

The reverse transcription reaction was performed by the PrimerScript ${ }^{\mathrm{TM}}$ RT reagent kit with gDNA Eraser (\#RR047A, Takara). qPCR was performed for selected IncRNAs by using PowerUp ${ }^{\text {TM }}$ SYBR $^{\text {TM }}$ Green Master Mix (REF A25742, applied biosystems by Thermo fisher scientific) with $2 \mu$ l cDNA. The primers used in this study were synthesized by TSINGKE (Biological Technology) and the sequences were listed in the supplemental table 2. PCR reactions were accomplished in QuantStudio 3 (applied biosystems by Thermo fisher scientific). Reactions were incubated in a 96-well optical plate following the manufacturer's instructions. Each sample was analyzed simultaneously in triplicate. Melting curve analysis was performed to validate the specific generation of the expected PCR product at the end of the PCR cycles. The expression changes of the IncRNAs were normalized to GAPDH, and the difference was calculated by the $2^{-\Delta \Delta \mathrm{Ct}}$ method [33]. The mean data of $\Delta \mathrm{Ct}$ for control groups was used as a calibrator $(\Delta \Delta \mathrm{Ct}=0,2-$ $\Delta \mathrm{Ct}=1)$.

\section{Statistical Analysis}

We used the interquartile range to find outliers. The differences between the 25th (quartile 1) and 75th percentiles (quartile 3) were used to identify extreme values (outliers) in the tails of the distribution. Statistical evaluation was performed by the Mann-Whitney $U$ test for means between patient and control groups and calculate by online statistical calculator (https://www.socscistatistics.com/).

\section{Prediction of IncRNAs function}

For the functional prediction of the IncRNAs, we adopted the methods mentioned in reference, first calculating the co-expressed mRNAs for each differentiated IncRNA, and then performing a functional enrichment analysis on this set of co-expressed mRNAs [34]. terms were used as the predicted functional term of given IncRNAs. Co-expressed mRNAs of IncRNAs with correlated P-values $<0.05$ were identified by calculating Pearson Correlation. We then used the hypergeometric cumulative distribution function to calculate the enrichment of the functional term in the annotations of co-expressed mRNAs. The false discovery rate was calculated following the reference [35].

After the intersection of the set of coding genes co-expressed by LncRNAs and the set of target genes of the transcription factor/chromatin regulatory complex is calculated, the hypergeometric distribution is used to calculate the enrichment degree of this intersection to obtain the transcription factors that are significantly associated with IncRNAs, thus identifying the transcription factors or chromatin regulatory factors that may play a regulatory role in combination with IncRNAs. By calculating the hypergeometric distribution, multiple IncRNA-TF relationship pairs can be obtained for each IncRNA. The Cytoscape software (http://www.cytoscape.org/) was used for network import and visualization. 


\section{Results}

\section{Microarray identification of dysregulated LncRNAs between ALS patients and healthy controls}

The microarray containing the 92727 probes for human coding and non-coding RNAs were used for screening dysregulation of RNAs for 5 SALS patients and 5 healthy control samples. The top 30 IncRNAs that differed the most from healthy controls were selected and expression discrepancy was presented as a heatmap (Fig. 1) by using Heatmapper software [36]. We used the microarray results to compare several different IncRNA databases, including LNCipedia, NONCODE and Annolnc [37-39]. Next, we named these transcripts with IDs corresponding to those in these databases. As shown in the heatmap, a total of 16 IncRNAs are down-regulating, and the remaining 14 IncRNAs are up-regulating in peripheral leukocytes of SALS comparing with controls.

\section{Verification and analysis of IncRNAs regulation by Quantitative Real-Time PCR}

To confirm microarray results, we performed reverse transcription-quantitative real-time PCR (RT-qPCR) for several selected IncRNAs. We excluded the IncRNAs whose sequence is highly overlapping with mRNAs, and the remaining IncRNAs whose sequence is not overlapping or partially overlapping with mRNAs were validated by RT-qPCR. The cDNAs were accomplished from peripheral blood leukocytes of 52 SALS patients, 40 PD patients and 38 healthy controls. A total of 13 IncRNAs were verified in this study (Fig. 2 and Supplemental Fig. 1).

Among these IncRNAs, 6 IncRNA transcripts were exhibited expression discrepancy in the SALS case comparing with healthy controls. All of these transcripts showed down-regulation results in SALS cases: Inc-CNTN4-2:1, Inc-NR3C2-8:1, Inc-ABCA12-3:1, Inc-DYRK2-7:1, Inc-POTEM-4:7 and XIST (in male cases). Since XIST is usually located on the $X$ chromosome, the changes in XIST expression were compared by gender [40]. The results of Inc-DYRK2-7:1, Inc-ABCA12-3:1, Inc-POTEM-4:7 and XIST are opposite to those of microarray in SALS, and the remaining IncRNA results are in agreement with microarray. By comparing the samples in the microarray, we found that two of the SALS clinical samples were identical to those used for qPCR: SALS2 and SALS4 numbered in the microarray. The expression of Inc-DYRK2-7:1, IncABCA12-3:1 and Inc-POTEM-4:7 are both upregulated in qPCR results for sample SALS2, which are consistent with those of the microarray. However, the expression of XIST is downregulated in qPCR results for SALS2. Also, the qPCR results for these IncRNAs of SALS4 were exactly opposite to those of SALS, which are inconsistent with the microarray results. We speculate that some qPCR-validated regulation results are inconsistent with the microarray due to individual patient differences affecting the differential expression of IncRNAs in peripheral blood leukocytes.

To confirm whether the 6 IncRNAs selected by qPCR can only be used as biomarkers for SALS or also for other neurodegenerative diseases, we simultaneously used peripheral blood leukocyte samples from 40 
patients with Parkinson's disease as one of the controls for neurodegenerative diseases. In these PD samples, we found that some of the above IncRNAs had the same trend of differential expression. LncCNTN4-2:1, Inc-NR3C2-8:1 and XIST (male cases) were showed down-regulated in PD. However, IncCNTN4-2:1 and XIST (male cases) were more significantly reduced in PD relative to ALS and Inc-NR3C28:1 still showed reduced expression in PD but not more so than in ALS. A trend of elevated expression of Inc-DYRK2-7:1 and Inc-ABCA12-3:1 were observed only in PD, which is the opposite of SALS. The IncPOTEM-4:7, whose expression was reduced in SALS, were not differentially expressed in PD. XIST in female cases was only upregulated in PD but not in SALS.

Based on the above screening results in HC, SALS and PD, we know that Inc-DYRK2-7:1, Inc-POTEM-4:7 and Inc-ABCA12-3:1 are expressed down-regulation only in SALS, while Inc-CNTN4-2:1, Inc-NR3C2-8:1 and XIST (male cases) are expressed down-regulation in both SALS and PD leukocytes.

\section{Pathway analysis of IncRNAs}

The GO (Gene Oncology) term enrichment and KEGG (Kyoto Encyclopedia of Genes and Genomes) pathway analysis of SALS patients compared to healthy controls has been performed separately for IncRNAs with the differential expression as shown in the microarray test, in which TOP 10 enriched items were expressed (Fig. 3). the enriched GO for cellular components include the messenger ribonucleoprotein complex and nucleus (Fig. 3A). In terms of molecular function, the highest enrichment of GO terms targeted by differentially expressed IncRNAs was with respect to the activity of various transcription factors (Fig. 3B). Also, the highest enrichment of GO terms for the biological process targeted by differentially expressed IncRNAs included cellular ketone body metabolic process and mitotic chromosome condensation (Fig. 3C). Meanwhile, differentially expressed IncRNAs enriched in the KEGG pathway also included synthesis and degradation of ketone bodies, insulin signaling pathway, and TNF signaling pathway, in addition to various other proteins functional expression pathway (Fig. 3D).

The intersection of the set of coding genes co-expressed by IncRNAs with the set of target genes of transcription factors is calculated, and the enrichment of this intersection is calculated using the hypergeometric distribution to obtain transcription factors that are significantly associated with IncRNAs, thus identifying transcription factors that may play a regulatory role together with IncRNAs. The analysis yields multiple IncRNA, coding genes and transcription factor relationship pairs for each IncRNA. For the correspondence between IncRNAs and transcription factors that showed differential expression in the microarray results, the top 30 transcription factors with higher frequencies were selected to draw visual network diagrams with the associated target genes (Fig. 4). In the ternary network diagram, it was confirmed that Inc-ABCA12-3 and Inc-CNTN4-2, which were differentially expressed in the peripheral blood leukocytes of SALS patients, were directly related to several target genes and indirectly related to two transcription factors, ZNF263 and SIX5 (Fig. 4A). Additionally, there are also coding gene that are indicated in the predicted results that may be associated with IncRNAs that also be highly interested in disease progression (e.g. SNCA, encoding alpha-synuclein; FBXW11, encoding member of F-box family). In contrast, in the binary network diagram several IncRNAs were found to be associated with other transcription factors (Fig. 4B). These also included Inc-NR3C2-8 and Inc-DYRK2-7, which were 
differentially expressed in peripheral blood leukocytes of SALS patients and were associated with the transcription factors ZBTB7A and BRCA1, respectively.

\section{Discussion}

Previous studies have found that the delay between the onset of ALS symptoms and diagnosis ranged up to 15.6 months [26]. Also, the patients with sporadic forms of the disease took relatively longer to diagnose than other patients[41]. Delays in diagnosis can affect the outcome as well as the survival of ALS patients, therefore, the discovery of an accurate and sensitive biomarker is critical to current ALS research. Since blood sampling has the advantages of easy handling and low-cost multiplex assays, biomarkers in the blood are our primary consideration for research. Besides, on the basis that $40 \%$ of IncRNAs are expressed in the human brain, it is a more promising potential biomarker for neurodegenerative diseases.

LncRNAs have been validated in recent studies of various disease pathways to be relevant to many biomolecular functions $[14,18]$. In this study, we have profiled IncRNAs in SALS patients as well as in healthy controls by microarray to expand our knowledge of molecular alterations in the transcriptome and obtain new data on their dysregulation. In the top 30 dysregulated IncRNAs, we validated 6 of them have differential expression in peripheral leukocytes of SALS comparing with healthy controls. We observed these 6 IncRNA transcripts have legible down-regulation (Inc-CNTN4-2:1, Inc-NR3C2-8:1, IncABCA12-3:1, Inc-DYRK2-7:1, Inc-POTEM-4:7 and XIST in male cases) in SALS cases. Total 3 IncRNAs (IncCNTN4-2:1, Inc-NR3C2-8:1 and XIST in male cases) showed the same trend of regulation but the differences are not expressed to the same extent in SALS and PD according to the P-values. Therefore, we believe that Inc-ABCA12-3:1, Inc-DYRK2-7:1 and Inc-POTEM-4:7 can be considered as SALS-specific peripheral blood biomarkers, while the other IncRNAs may serve as peripheral blood biomarkers for neurodegenerative diseases. It is also worth mentioning that other studies have shown that the use of peripheral blood for ALS-associated biomarker screening is a good foundation to pave the way for ALSassociated RNA function studies [28]. It is still unfortunate that for most of the remaining IncRNA sequences that overlap highly or completely with the mRNA sequences, we are currently unable to have a more suitable method to validate their expression in clinical samples.

Substantial evidence reveals that RNA as a key regulator associated with other ALS relative proteins plays an important role in ALS relevant RNA metabolism as well as in the transcription of other proteins[5, $6,42,43]$. Among these disease related RNAs, two main RNA families can be distinguished, coding RNAs and non-coding RNAs. They are both associated with RNA metabolism and can produce cellular defects that can be the cause of ALS. Alteration of numerous IncRNAs has been described in different types of motor neuron diseases, however only very few of them have been subjected to in-depth studies of functions related to disease mechanisms [44]. The paraspeckle, a distinct cellular feature of ALS, was revealed that its formation connected with IncRNA NEAT1_2 which was reported association with ALS $[29,45]$. This IncRNA has been shown directly bound to TDP-43 and FUS, which are the proteins enriched in paraspeckles [29]. This is one of the most in-depth studies targeting the mechanism of IncRNA in ALS, 
while our data will provide more evidence and ideas for studies on IncRNA as a biomarker in ALS and related mechanisms.

What has attracted the attention of researchers in recent years is the variation and significance of its interaction with miRNAs or mRNAs and the networks they associate with various diseases [46-48]. Through bioinformatics analysis, we found that a significant proportion of IncRNAs with differential expression in microarray and peripheral blood leukocyte screens were associated with various signaling pathways and transcription factors. In the GO component analysis, we obtained results similar to those in the literature, with differentially expressed IncRNAs being most associated with the messenger ribonucleoprotein complex in cellular components which is closely related to mRNA. In GO molecular function and biological process analysis, the items most associated with differentially expressed IncRNAs: 3-oxoacid CoA-transferase and cellular ketone body metabolic process are both strongly associated with fatty acid metabolism, and their deficiency is expressed in the development of multiple diseases[49-52]. Also, some findings suggest that unsaturated fatty acid metabolism is significantly dysregulated in the brains of patients with neurodegenerative diseases [53, 54]. Furthermore, among the predicted results of IncRNA-associated coding gene and transcription factor, SNCA (encoding alphasynuclein), FBXW11 (encoding F-box family member) and BRCA1 (DNA repair-associated) may be associated with neurodegenerative diseases [55-57]. Therefore, we believe that the results of this bioinformatic analysis of IncRNAs are consistent with other clinical findings and can be used in the future as an idea to study the mechanism and treatment of IncRNAs related to ALS.

LncRNAs can regulate their molecular functions as well as those of other molecules in the cytoplasm or nucleus through a variety of mechanisms [18]. Thus, for differential expressed IncRNAs it is also necessary to perform intracellular localization experiments in multiple cell lines. The intracellular localization of IncRNAs is highly relating to their association with other molecules which is similar to mRNA [58]. XIST, a IncRNA named X-inactive specific transcript, has been intensively expounded in multiple studies in recent years. The intracellular localization of XIST has been confirmed in the nucleus [59]. Correlation of XIST with functions and mechanisms studied in the nucleus has also been confirmed: association with silencing transcription, inducing chromatin formation and diacylation $[60,61]$. In this trial XIST was downregulated in the peripheral blood leukocytes of male SALS and PD patients, thus more studies on the mechanisms and functions of neurodegenerative diseases associated with XIST need to be demonstrated as soon as possible [62]. The other IncRNAs reported in this study serve as novel biomarkers that need to be predicted and validated for their ALS-related mechanisms and functions based on their respective intracellular localization. Most IncRNAs were validated for their association with mRNAs or miRNAs after they were verified for anomalous change in expression in the presence of disease. Numerous results illustrate that IncRNA-mRNA-miRNA pathway studies can indeed provide conjecture and basis for our understanding of the functional mechanisms of IncRNAs [46, 47, 63-65]. However, only a few ceRNA interactions have been studied concerning neurodegenerative diseases. Nevertheless, we are still interested in IncRNA-miRNA interactions in neurodegenerative diseases and will continue to better understand this mechanism in subsequent studies. Besides, our bioinformatics data 
indicates the possible relation between dysregulated IncRNAs and their target genes or relative transcription factors.

\section{Conclusion}

In summary, our study elucidates the expression discrepancy and intracellular characteristics of IncRNAs in peripheral blood leukocytes from SALS patients, which may provide new SALS biomarkers for clinical diagnosis. The bioinformatic analysis of IncRNAs with expression discrepancy also provides information for subsequent functional studies on these IncRNAs.

\section{Abbreviations}

ALS, Amyotrophic lateral sclerosis

SALS, sporadic amyotrophic lateral sclerosis

PD, Parkinson's disease NcRNAs, non-coding RNAs

LncRNAs, long non-coding RNAs

RT-qPCR, reverse transcription-quantitative real-time PCR

GO, Gene Oncology

KEGG, Kyoto Encyclopedia of Genes and Genomes

$\mathrm{TF}$, transcription factors

\section{Declarations}

\section{Ethics approval and consent to participate}

West China Hospital approved the study, and all participants gave signed informed consent.

\section{Consent for publication}

Not applicable.

\section{Availability of data and materials}

All data generated or analyzed during this study are included in this article and its supplementary materials.

\section{Competing interests}


The authors declare that they have no competing interests.

\section{Funding}

The present study was supported by the funding of The National Natural Science Foundation of China (Grant No. 81871000).

\section{Authors' contributions}

YJY searched and selected the studies, analyzed the data, drafted and revised the article. DJP, XJG, YPC, RWO and QQW prepared the samples and gave suggestions on study design. CYL and HFS designed the study and gave suggestions on revising the article. All authors read and approved the final manuscript.

\section{Acknowledgements}

The authors thank all subjects for their participation in the study.

\section{References}

1. Kurland LT, Mulder DW (1955) Epidemiologic investigations of amyotrophic lateral sclerosis. 2. Familial aggregations indicative of dominant inheritance. I Neurology 5:182-196

2. Camu W, Khoris J, Moulard B et al (1999) Genetics of familial ALS and consequences for diagnosis. J Neurol Sci 165:S21-S26

3. Kiernan MC, Vucic S, Cheah BC et al (2011) Amyotrophic lateral sclerosis. Lancet 377:942-955

4. Taylor JP, Brown RH, Cleveland DW (2016) Decoding ALS: from genes to mechanism. Nature 539:197-206

5. Morgan S, Orrell RW (2016) Pathogenesis of amyotrophic lateral sclerosis. Br Med Bull 119:87-97

6. Krokidis MG, Vlamos P (2018) Transcriptomics in amyotrophic lateral sclerosis. Frontiers in Bioscience - Elite 10:103-121

7. Van Deerlin VM, Leverenz JB, Bekris LM et al (2008) TARDBP mutations in amyotrophic lateral sclerosis with TDP-43 neuropathology: a genetic and histopathological analysis. Lancet Neurol 7:409-416

8. Mackenzie IR, Rademakers R, Neumann M (2010) TDP-43 and FUS in amyotrophic lateral sclerosis and frontotemporal dementia. Lancet Neurol 9:995-1007

9. Greenway MJ, Andersen PM, Russ C et al (2006) ANG mutations segregate with familial and "sporadic" amyotrophic lateral sclerosis. Nat Genet 38:411-413

10. DeJesus-Hernandez M, Mackenzie IR, Boeve BF et al (2011) Expanded GGGGCC hexanucleotide repeat in noncoding region of C9ORF72 causes chromosome 9p-linked FTD and ALS. Neuron 72:245-256 
11. ENCODE Project Consortium (2012) An integrated encyclopedia of DNA elements in the human genome. Nature 489:57-74

12. Gomes AQ, Nolasco S, Soares H (2013) Non-coding RNAs: multi-tasking molecules in the cell. Int J Mol Sci 14:16010-16039

13. Harries LW (2012) Long non-coding RNAs and human disease. Biochem Soc Trans 40:902-906

14. Adams BD, Parsons $C$, Walker $L$ et al (2017) Targeting noncoding RNAs in disease. Journal of Clinical Investigation 127:761-771

15. Kiss T (2004) Biogenesis of small nuclear RNPs. J Cell Sci 117:5949-5951

16. Rinn JL, Chang HY (2012) Genome regulation by long noncoding RNAs. Annu Rev Biochem 81:145166

17. Ulitsky I, Bartel DP (2013) lincRNAs: genomics, evolution, and mechanisms. Cell 154:26-46

18. Atianand MK, Caffrey DR, Fitzgerald KA (2017) Immunobiology of Long Noncoding RNAs. Annu Rev Immunol 35:177-198

19. Derrien T, Johnson R, Bussotti G et al (2012) The GENCODE v7 catalog of human long noncoding RNAs: Analysis of their gene structure, evolution, and expression. Genome Res 22:1775-1789

20. Zhong J, Chuang SC, Bianchi R et al (2009) BC1 regulation of metabotropic glutamate receptormediated neuronal excitability. J Neurosci 29:9977-9986

21. Wang A, Wang J, Liu Y, Zhou Y (2017) Mechanisms of long non-coding RNAs in the assembly and plasticity of neural circuitry. Front Neural Circuits 11:76

22. Choi J, Levey Al, Weintraub ST et al (2004) Oxidative Modifications and Down-regulation of Ubiquitin Carboxyl-terminal Hydrolase L1 Associated with Idiopathic Parkinson's and Alzheimer's Diseases. J Biol Chem 279:13256-13264

23. Yang B, Xia Z an, Zhong B et al (2017) Distinct Hippocampal Expression Profiles of Long Non-coding RNAs in an Alzheimer's Disease Model. Mol Neurobiol 54:4833-4846

24. Yan W, Chen ZY, Chen JQ, Chen HM (2018) LncRNA NEAT1 promotes autophagy in MPTP-induced Parkinson's disease through stabilizing PINK1 protein. Biochem Biophys Res Commun 496:10191024

25. Feng L, Liao Y-T, He J-C et al (2018) Plasma long non-coding RNA BACE1 as a novel biomarker for diagnosis of Alzheimer disease. BMC Neurol 18:4

26. Cellura E, Spataro R, Taiello AC, La Bella V (2012) Factors affecting the diagnostic delay in amyotrophic lateral sclerosis. Clin Neurol Neurosurg 114:550-554

27. Vejux A, Namsi A, Nury T et al (2018) Biomarkers of Amyotrophic Lateral Sclerosis: Current Status and Interest of Oxysterols and Phytosterols. Frontiers in Molecular Neuroscience 11:12

28. Gagliardi S, Zucca S, Pandini C et al (2018) Long non-coding and coding RNAs characterization in Peripheral Blood Mononuclear Cells and Spinal Cord from Amyotrophic Lateral Sclerosis patients. Sci Rep 8:2378 
29. Nishimoto Y, Nakagawa S, Hirose T et al (2013) The long non-coding RNA nuclear-enriched abundant transcript 1-2 induces paraspeckle formation in the motor neuron during the early phase of amyotrophic lateral sclerosis. Mol Brain 6:31

30. Wei Q, Chen X, Zheng Z et al (2015) Clinical features of amyotrophic lateral sclerosis in south-west China. Amyotroph Lateral Scler Frontotemporal Degener 16:512-519

31. Renton AE, Majounie E, Waite A et al (2011) A hexanucleotide repeat expansion in C9ORF72 is the cause of chromosome 9p21-linked ALS-FTD. Neuron 72:257-268

32. Chen $Y$, Lin Z, Chen $X$ et al (2016) Large C9orf72 repeat expansions are seen in Chinese patients with sporadic amyotrophic lateral sclerosis. Neurobiol Aging 38:217.e15-217.e22

33. Livak KJ, Schmittgen TD (2001) Analysis of relative gene expression data using real-time quantitative PCR and the 2- $\Delta \Delta C T$ method. Methods 25:402-408

34. Guttman M, Amit I, Garber M et al (2009) Chromatin signature reveals over a thousand highly conserved large non-coding RNAs in mammals. Nature 458:223-227

35. Storey JD (2002) A direct approach to false discovery rates. J R Stat Soc Series B Stat Methodol 64:479-498

36. Babicki S, Arndt D, Marcu A et al (2016) Heatmapper: web-enabled heat mapping for all. Nucleic Acids Res 44:W147-W153

37. Volders PJ, Anckaert J, Verheggen K et al (2019) Lncipedia 5: Towards a reference set of human long non-coding rnas. Nucleic Acids Res 47:D135-D139

38. Fang S, Zhang L, Guo J et al (2018) NONCODEV5: A comprehensive annotation database for long non-coding RNAs. Nucleic Acids Res 46:D308-D314

39. Hou M, Tang X, Tian F et al (2016) AnnoLnc: A web server for systematically annotating novel human IncRNAs. BMC Genom 17:931

40. Brown CJ, Hendrich BD, Rupert JL et al (1992) The human XIST gene: Analysis of a $17 \mathrm{~kb}$ inactive Xspecific RNA that contains conserved repeats and is highly localized within the nucleus. Cell 71:527542

41. Paganoni S, Macklin EA, Lee A et al (2014) Diagnostic timelines and delays in diagnosing amyotrophic lateral sclerosis (ALS). Amyotroph Lateral Scler Frontotemporal Degener 15:453-456

42. Gagliardi S, Milani P, Sardone V et al (2012) From Transcriptome to Noncoding RNAs: Implications in ALS Mechanism. Neurology Research International 2012:278725

43. Strong MJ (2010) The evidence for altered RNA metabolism in amyotrophic lateral sclerosis (ALS). J Neurol Sci 288:1-12

44. Chen K-W, Chen J-A (2020) Functional Roles of Long Non-coding RNAs in Motor Neuron Development and Disease. J Biomed Sci 27:38

45. Hutchinson JN, Ensminger AW, Clemson CM et al (2007) A screen for nuclear transcripts identifies two linked noncoding RNAs associated with SC35 splicing domains. BMC Genom 8:39 
46. Ma N, Tie C, Yu B et al (2020) Identifying IncRNA-miRNA-mRNA networks to investigate Alzheimer's disease pathogenesis and therapy strategy. Aging 12:2897-2920

47. Tang X, Feng D, Li M et al (2019) Transcriptomic Analysis of mRNA-IncRNA-miRNA Interactions in Hepatocellular Carcinoma. Sci Rep 9:1-12

48. Zhang Y, Li Y, Wang Q et al (2017) Identification of an incRNA-miRNA-mRNA interaction mechanism in breast cancer based on bioinformatic analysis. Mol Med Rep 16:5113-5120

49. Wakil SJ, Abu-Elheiga LA (2009) Fatty acid metabolism: target for metabolic syndrome. J Lipid Res 50 Suppl:S138-S143

50. Turko IV, Marcondes S, Murad F (2001) Diabetes-associated nitration of tyrosine and inactivation of succinyl-CoA:3-oxoacid CoA-transferase. Am J Physiol Heart Circ Physiol 281:H2289-H2294

51. Cotter DG, Schugar RC, Crawford PA (2013) Ketone body metabolism and cardiovascular disease. Am J Physiol Heart Circ Physiol 304:H1060-H1076

52. Fukao T, Mitchell G, Sass JO et al (2014) Ketone body metabolism and its defects. In: Journal of Inherited Metabolic Disease. Kluwer Academic Publishers, pp 541-551

53. Snowden SG, Ebshiana AA, Hye A et al (2017) Association between fatty acid metabolism in the brain and Alzheimer disease neuropathology and cognitive performance: A nontargeted metabolomic study. PLoS Med 14:e1002266

54. Bogie JFJ, Haidar M, Kooij G, Hendriks JJA (2020) Fatty acid metabolism in the progression and resolution of CNS disorders. Adv Drug Deliv Rev 159:198-213

55. Bernal-Conde LD, Ramos-Acevedo R, Reyes-Hernández MA et al (2019) Alpha-Synuclein Physiology and Pathology: A Perspective on Cellular Structures and Organelles. Frontiers in Neuroscience 13:1399

56. Rayner SL, Morsch M, Molloy MP et al (2019) Using proteomics to identify ubiquitin ligase-substrate pairs: how novel methods may unveil therapeutic targets for neurodegenerative diseases. Cell Mol Life Sci 76:2499-2510

57. Noristani HN, Sabourin JC, Gerber YN et al (2015) Brca1 is expressed in human microglia and is dysregulated in human and animal model of ALS. Mol Neurodegener 10:34

58. Cabili MN, Dunagin MC, McClanahan PD et al (2015) Localization and abundance analysis of human IncRNAs at single-cell and single-molecule resolution. Genome Biol 16:20

59. Clemson CM, McNeil JA, Willard HF, Lawrence JB (1996) XIST RNA paints the inactive X chromosome at interphase: Evidence for a novel RNA involved in nuclear/chromosome structure. $J$ Cell Biol 132:259-275

60. McHugh CA, Chen CK, Chow A et al (2015) The Xist IncRNA interacts directly with SHARP to silence transcription through HDAC3. Nature 521:232-236

61. Cerase A, Armaos A, Cid F et al (2018) Xist IncRNA forms silencing granules that induce heterochromatin formation and repressive complexes recruitment by phase separation. bioRxiv 351015 
62. Cerase A, Armaos A, Neumayer C et al (2019) Phase separation drives X-chromosome inactivation: a hypothesis. Nature Structural Molecular Biology 26:331-334

63. Kallen AN, Zhou XB, Xu J et al (2013) The Imprinted H19 LncRNA Antagonizes Let-7 MicroRNAs. Mol Cell 52:101-112

64. Wang $Y, X u Z$, Jiang $J$ et al (2013) Endogenous miRNA Sponge lincRNA-RoR Regulates Oct4, Nanog, and Sox2 in Human Embryonic Stem Cell Self-Renewal. Dev Cell 25:69-80

65. Tay Y, Rinn J, Pandolfi PP (2014) The multilayered complexity of ceRNA crosstalk and competition. Nature 505:344-352

\section{Figures}

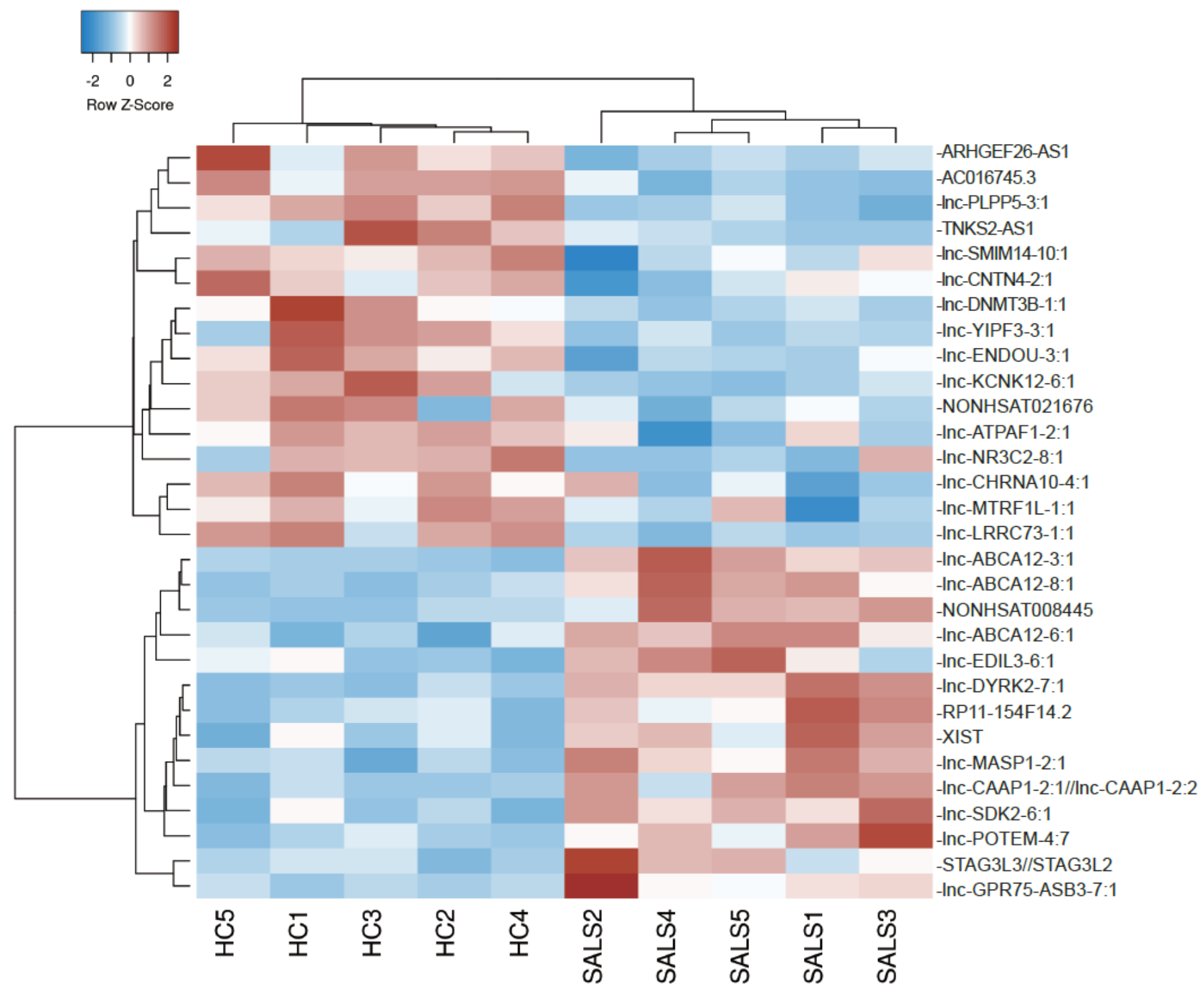

Figure 1 
Microarray results are represented by hierarchical clustering analysis heatmap Microarray expression heatmap of differently expressed IncRNAs in ALS and healthy controls. In this panel differentially 30 expressed IncRNAs are shown. All comparisons are given between the disease state and the control samples. Average linkage and Pearson distance measurement were performed for clustering.
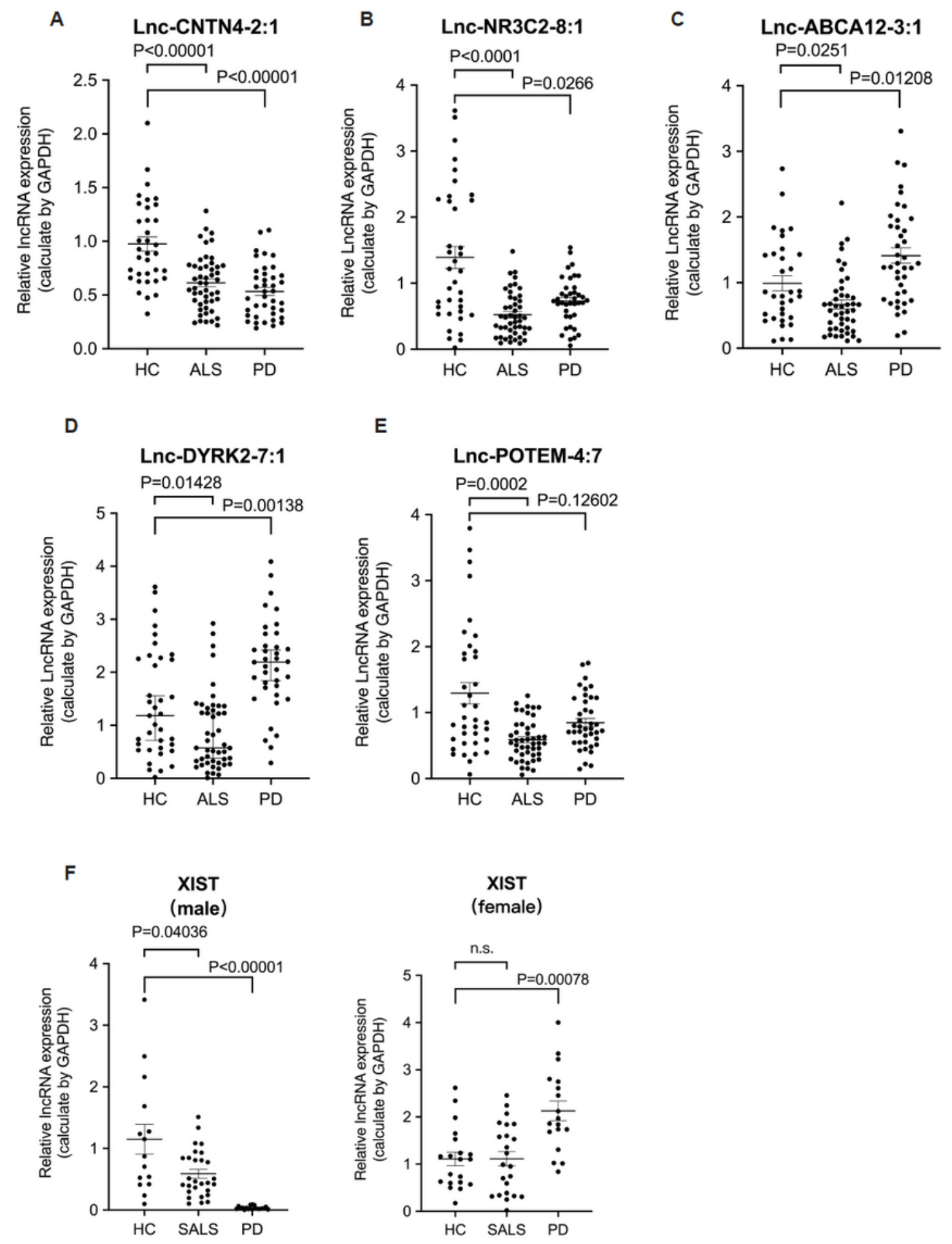

Figure 2 
Dysregulation of selected IncRNAs in SALS and PD patients Differentially expressed transcripts verified by Real-Time PCR in peripheral leukocytes from SALS, PD and healthy controls (HC). (A) Inc-CNTN4-2:1; (B) Inc-NR3C2-8:1; (C) Inc-ABCA12-3:1; (D) Inc-DYRK2-7:1; (E) Inc-POTEM-4:7; (F) XIST in gender subgroups. $p$ values were calculated using two-tailed Mann-Whitney $U$ test. Horizontal bars show mean \pm SEM. In the main content, the group in which the transcript was found deregulated is reported. The extreme values have been eliminated from the graph.

A

GO component analysis

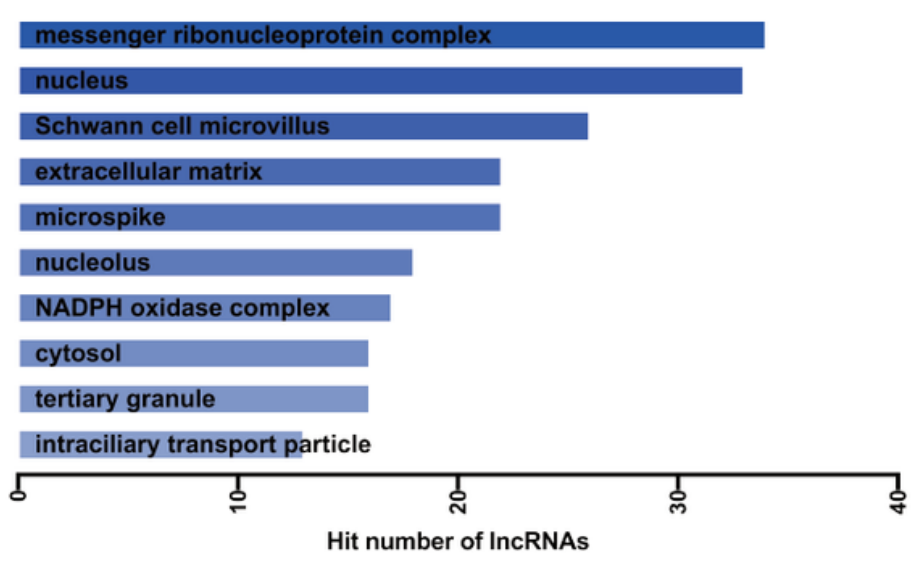

C

GO process analysis

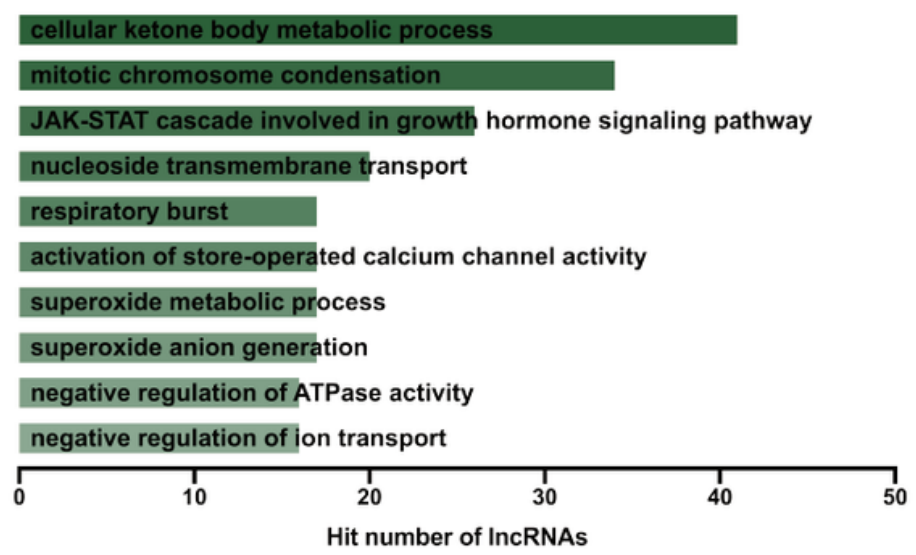

B

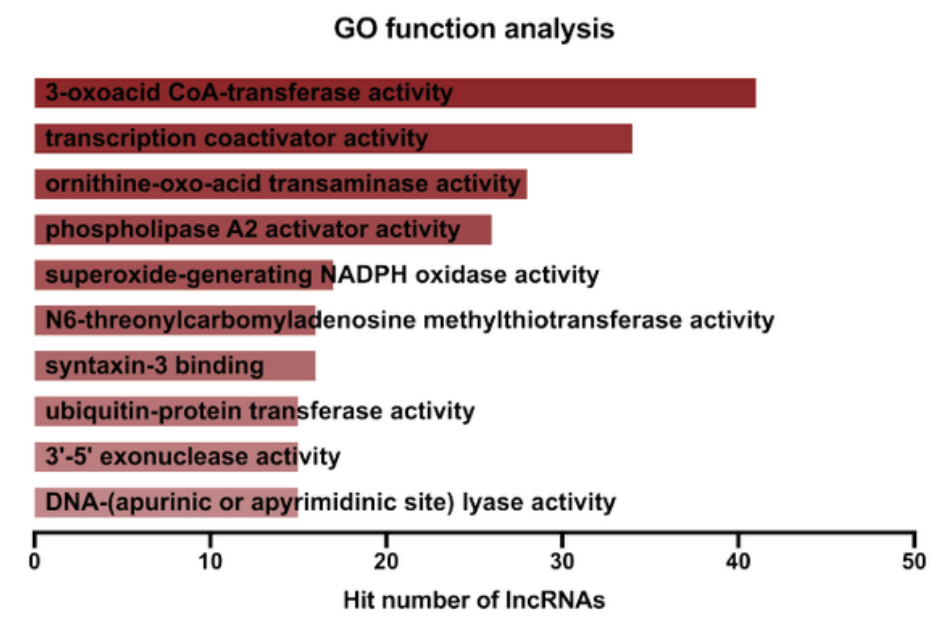

D

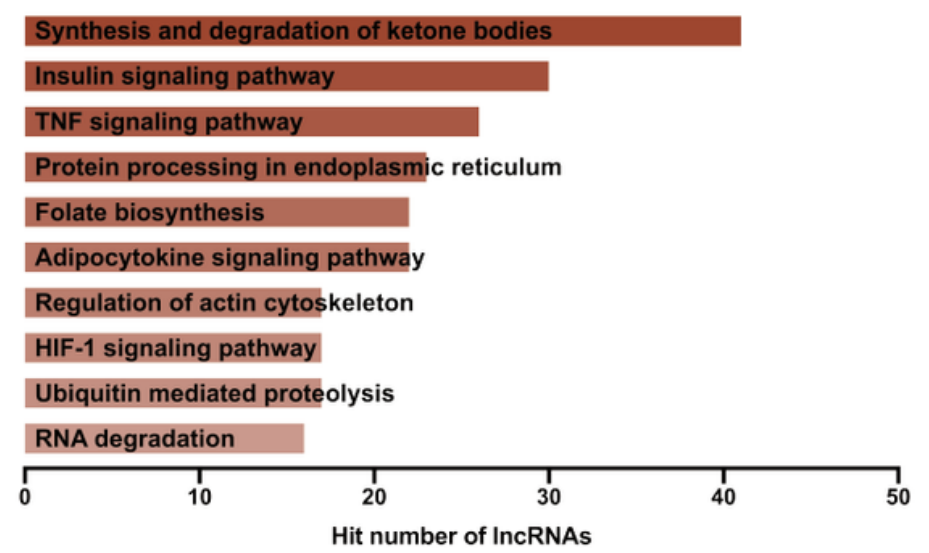

\section{Figure 3}

GO analysis and KEGG pathway analysis for differential expressed IncRNAs of microarray in SALS patients compared to healthy controls TOP 10 enriched GO terms for (A) cellular component; (B) molecular function; and (C) biological process. (D) TOP 10 enriched KEGG pathways of differential expressed IncRNAs from microarray. The length of the bar represents the hit number of differentials expressed IncRNAs from the microarray. 
A
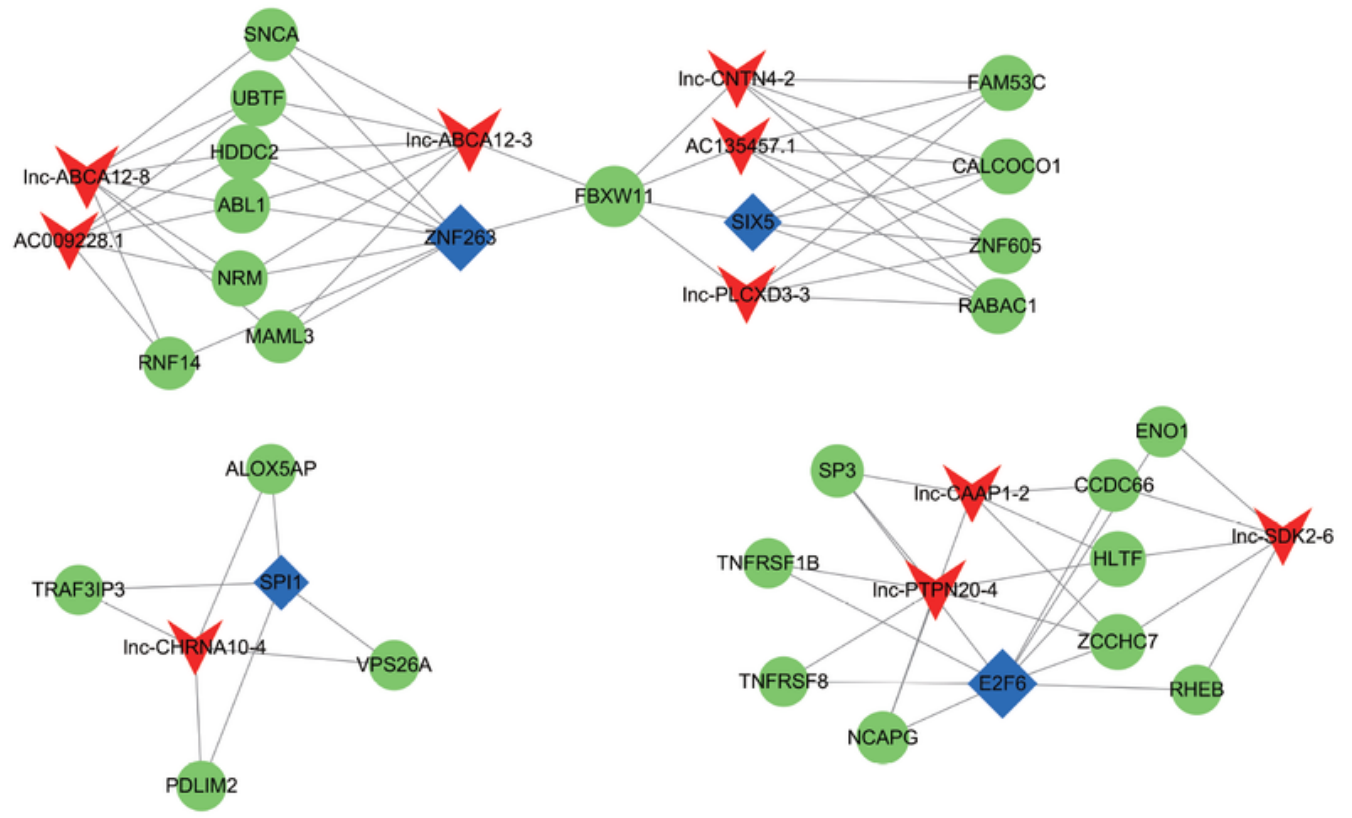

B

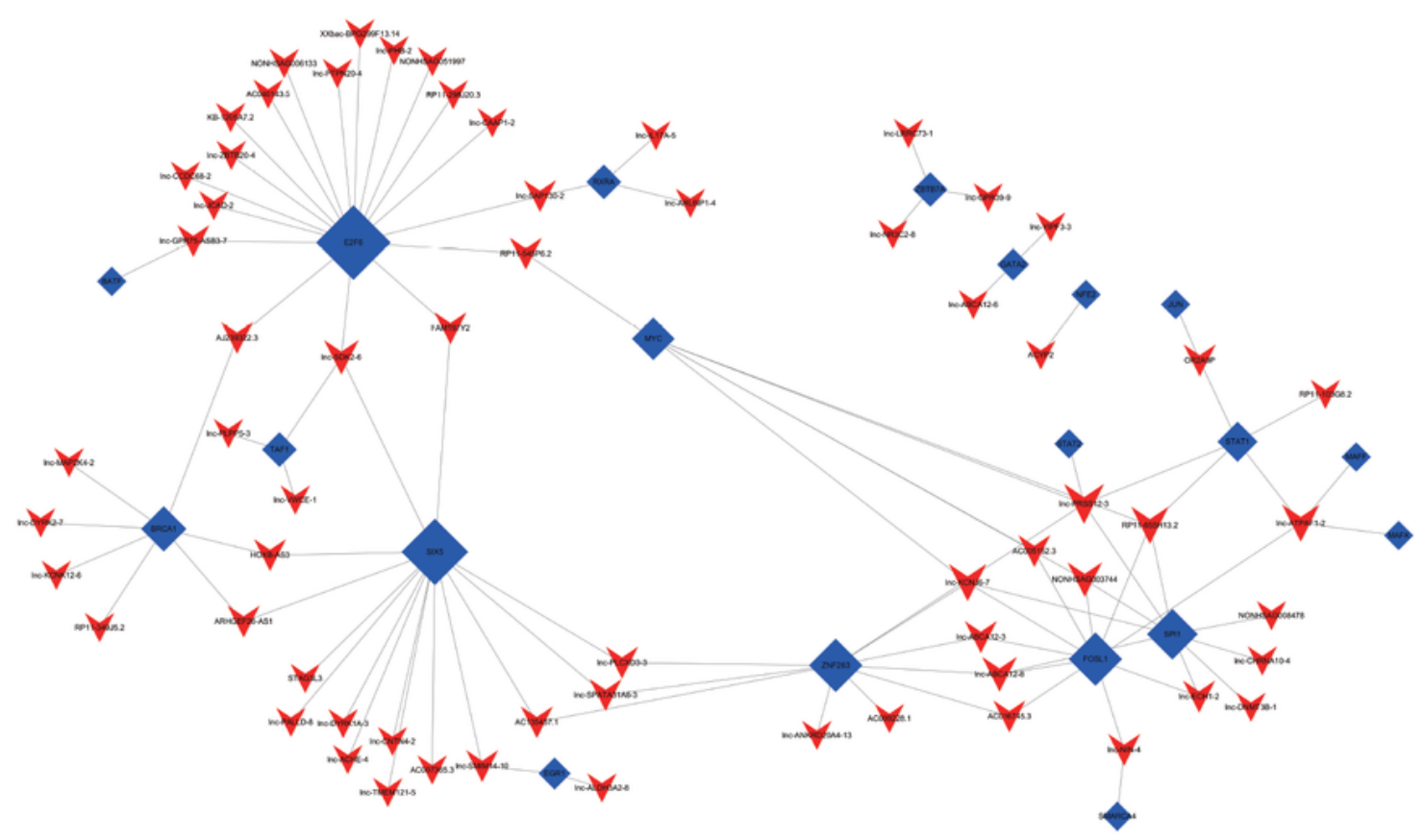

\section{Figure 4}

Coding non-coding RNAs co-expression analysis The network represents co-expression correlations between mRNAs, IncRNAs and transcription factors. (A) Ternary network diagrams of IncRNAs, transcription factors and mRNAs. (B) Binary network diagrams of IncRNAs and transcription factors. Red triangular arrows refer to differentially expressed IncRNAs, green dots refer to the target gene, blue squares refer to transcription factors. 


\section{Supplementary Files}

This is a list of supplementary files associated with this preprint. Click to download.

- supplementarymaterials.pdf 